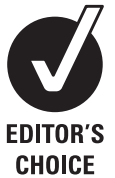

CHOICE

\begin{abstract}
'The Heart Hospital, London, UK ${ }^{2}$ Institute of Cardiovascular Science, University College London, London, UK ${ }^{3}$ Centre for Amyloidosis and Acute Phase Proteins and National Amyloidosis Centre, University College London, London, UK

${ }^{4}$ The National Hospital for Neurology and Neurosurgery, Charles Dent Metabolic Unit, London, UK

${ }^{5}$ The Royal Free Hospital Lysosomal Storage Disorders Unit, Royal Free Hospital, London, UK

${ }^{6}$ Cardiovascular Unit, UCL Institute of Child Health, London, UK
\end{abstract}

\section{Correspondence to} Dr James Moon, The Heart Hospital, 16-18 Westmoreland St, London W1G 8PH, UK james.moon@uclh.nhs.uk

Accepted 14 June 2012 Published Online First 30 August 2012

\title{
Cardiovascular magnetic resonance measurement of myocardial extracellular volume in health and disease
}

\author{
Daniel M Sado, ${ }^{1,2}$ Andrew S Flett, ${ }^{1,2}$ Sanjay M Banypersad, ${ }^{1,2,3}$ Steven K White, ${ }^{1,2}$ \\ Viviana Maestrini, ${ }^{1}$ Giovanni Quarta, ${ }^{1}$ Robin H Lachmann, ${ }^{4}$ Elaine Murphy, ${ }^{4}$ \\ Atul Mehta, ${ }^{5}$ Derralynn A Hughes, ${ }^{5}$ William J McKenna, ${ }^{1,2}$ Andrew M Taylor, ${ }^{2,6}$ \\ Derek J Hausenloy, ${ }^{1,2}$ Philip N Hawkins, ${ }^{3}$ Perry M Elliott, ${ }^{1,2}$ James C Moon ${ }^{1,2}$
}

\section{ABSTRACT}

Objective To measure and assess the significance of myocardial extracellular volume (ECV), determined noninvasively by equilibrium contrast cardiovascular magnetic resonance, as a clinical biomarker in health and a number of cardiac diseases of varying pathophysiology. Design Prospective study.

Setting Tertiary referral cardiology centre in London, UK. Patients 192 patients were mainly recruited from specialist clinics. We studied patients with Anderson-Fabry disease (AFD, $n=17$ ), dilated cardiomyopathy (DCM, $n=31$ ), hypertrophic cardiomyopathy (HCM, $n=31$ ), severe aortic stenosis (AS, $n=66)$, cardiac AL amyloidosis $(n=27)$ and myocardial infarction ( $\mathrm{Ml}, \mathrm{n}=20$ ). The results were compared with those for 81 normal subjects.

Results In normal subjects, ECV (mean (95\% Cl), measured in the septum) was slightly higher in women than men 10.273 (0.264 to 0.282 vs 0.233 (0.225 to $0.244), p<0.001)$, with no change with age. In disease, the ECV of AFD was the same as in normal subjects but higher in all other diseases $(p<0.001)$. Mean ECV was the same in DCM, HCM and AS $(0.280,0.291,0.276$ respectively), but higher in cardiac $A \mathrm{~L}$ amyloidosis and higher again in $\mathrm{Ml}(0.466$ and 0.585 respectively, each $p<0.001)$. Where ECV was elevated, correlations were found with indexed left ventricular mass, end systolic volume, ejection fraction and left atrial area in apparent disease-specific patterns.

Conclusions Myocardial ECV, assessed non-invasively in the septum with equilibrium contrast cardiovascular magnetic resonance, shows gender differences in normal individuals and disease-specific variability. Therefore, ECV shows early potential to be a useful biomarker in health and disease.

\section{INTRODUCTION}

The ratio of cell to extracellular volume (ECV) is a property of normal organs and tissues, which may change in disease through cell hypertrophy, hyperplasia, loss and/or extracellular expansion. ${ }^{1}$ Extracellular expansion consists of increased water, usually associated with increased collagen in focal or diffuse fibrosis, ${ }^{2}{ }^{3}$ or deposition of pathological material such as amyloid. ${ }^{4}$ Its quantification provides prognostic information about organ function, ${ }^{56}$ and may guide therapy, for example, in cirrhosis, $^{7}$ transplantation rejection ${ }^{8}$ and amyloidosis. ${ }^{9}$ In most cases, this information can only be obtained from invasive biopsy. Myocardial extracellular expansion occurs in most cardiac diseases, but clinical applications have been limited because of the hazard and sampling error of myocardial biopsy. $^{10}$ Cardiovascular magnetic resonance (CMR) can detect focal but not diffuse fibrosis using the late gadolinium enhancement technique. ${ }^{11}$ Chelated gadolinium contrast is an extracellular contrast agent which visibly reduces the T1 relaxation time of tissue. After bolus intravenous administration, it distributes with slower kinetics and higher volume of distribution in focal fibrosis generating relative signal difference. If instead, T1 is measured directly (T1 mapping), mathematical modelling can potentially remove kinetic effects and derive the volume of distribution, reflecting ECV. $^{12}{ }^{13}$ Here, we have used a new approach, equilibrium contrast cardiovascular magnetic resonance (EQ-CMR), where a primed contrast infusion is used to eliminate contrast kinetic effects, ${ }^{14}$ to assess the myocardial ECV of normal subjects and patients with a range of cardiac diseases. We hypothesised that myocardial ECV would (a) be measurable across multiple diseases, (b) vary according to the underlying disease, and (c) correlate with other CMR markers of disease severity.

\section{METHODS}

The research received approval from the local research ethics committee, and all participants provided written informed consent. EQ-CMR, as previously validated against histology in aortic stenosis and hypertrophic cardiomyopathy, ${ }^{14}$ uses a conventional cardiac-enabled MRI scanner (1.5T Avanto; Siemens Medical Solutions, Erlangen, Germany) and is an add-on to a standard clinical scan. We quantified the tissue volume of distribution of gadoterate meglumine (gadolinium-DOTA, marketed as Dotarem by Guerbet, Roissy, France), which partitions freely between the plasma and extracellular space but does not enter cells. Extracellular tissue volume is primarily determined by the amount of extracellular matrix. EQ-CMR involves three steps: (1) a standard gadolinium bolus $(0.1 \mathrm{mmol} / \mathrm{kg})$ followed by a constant infusion $(0.002 \mathrm{mmol} / \mathrm{kg} / \mathrm{min})$ to eliminate contrast kinetic effects and achieve an equilibrium contrast state throughout the body; (2) T1 measurement before and after contrast equilibrium using CMR, here using a fast low-angle single-shot inversion 
recovery (FLASH IR) sequence at increasing inversion times from 140 to 900 to allow quantification of T1 in the fourchamber long-axis view; and (3) a direct measure of blood volume of contrast distribution, by taking a complete blood count, equating the blood volume of contrast distribution to one minus the haematocrit. The myocardial ECV is then calculated as $^{12}$ :

$$
\begin{aligned}
& \text { Myocardial ECV }= \\
& (1-\text { haematocrit }) \times \frac{(1 / \mathrm{T} 1 \mathrm{mpc}-1 / \mathrm{T} 1 \mathrm{MPC})}{(1 / \mathrm{T} 1 \mathrm{bpc}-1 / \mathrm{T} 1 \mathrm{bpc})}
\end{aligned}
$$

All normal subjects ( $n=81$, median age 43 (range $24-81$ ), 52\% male) were only recruited through advertising within the hospital, university, general practitioner surgeries and a local dancing class. None were referred as patients for a clinical CMR scan that then turned out to be normal. All normal subjects had no history or symptoms of cardiovascular disease or diabetes. Four subjects had been prescribed statin therapy for hypercholesterolaemia (primary cardiovascular prevention), but no other normal subject was using any cardiovascular medication. All subjects had a normal blood pressure (defined as $\leq 140$ / $90 \mathrm{~mm} \mathrm{Hg}$; mean systolic blood pressure, 124 12 ; mean diastolic blood pressure, 76 19 ), 12-lead ECG and clinical CMR scan. Normal subject baseline characteristics are outlined in table 1.

Patients ( $n=192$ ) were prospectively recruited with any one of six conditions:

1. Anderson-Fabry disease (AFD; $\mathrm{n}=17 ; 76 \%$ male; median age of men 43 (range 19-59); median age of women 52 (range 40-67)). All patients had a genetically confirmed diagnosis and were receiving enzyme replacement therapy. Eighty-eight per cent of patients (including all female patients) had left ventricular hypertrophy as assessed using CMR steady-state free precession cine short-axis views (elevated indexed left ventricular mass and wall thickness $>11 \mathrm{~mm}$ ). The mean indexed mass was $139 \mathrm{~g} / \mathrm{m}^{2}$ (range 84-230) in the male patients and $116 \mathrm{~g} / \mathrm{m}^{2}$ (range 98-129) in the female patients. The mean wall thickness was $14 \mathrm{~mm}$ in the male patients and $15 \mathrm{~mm}$ in the female patients. Fifty-three per cent of patients had late gadolinium enhancement (most commonly seen in the basal inferolateral wall).

2. Familial dilated cardiomyopathy (DCM; $n=31$; median age 41 (range 19-75); 82\% male). All patients met previously described diagnostic criteria. ${ }^{15}$ with most having mild disease expression (mean indexed left ventricular end diastolic volume $121 \%$ of predicted; mean CMR ejection fraction $53 \%$ ). Thirty-five per cent of patients had late gadolinium enhancement, mostly in mid wall of the basal to mid septum.

3. Hypertrophic cardiomyopathy $(\mathrm{HCM} ; \mathrm{n}=31$; median age 50 (range 19-74), 71\% male). All patients met previously described diagnostic criteria. ${ }^{16}$ Seventy-one per cent of patients had an asymmetrical septal hypertrophy pattern, with the remainder apical predominant hypertrophy. The mean maximal wall thickness (from CMR steady-state free precession short-axis assessment) was $20 \mathrm{~mm}$. Sixty-five per cent of patients had left ventricular outflow tract obstruction. Sixty-one per cent of patients were found to have late gadolinium enhancement in a variety of locations, such as at the right ventricular insertion points or the left ventricular apex.

4. Severe aortic stenosis (AS; $n=66$; median age 67 (range 32-86), 82\% male). All patients had undergone clinical evaluation and echocardiography for diagnosis (peak gradient $76 \mathrm{~mm} \mathrm{Hg}$, mean $47 \mathrm{~mm} \mathrm{Hg}$, mean valve area by continuity equation $0.75 \mathrm{~cm}^{2}$ ) and were listed for surgical aortic valve replacement. Forty-two per cent of patients had late gadolinium enhancement in a variety of locations.

5. Cardiac AL amyloidosis ( $n=27$; median age 66 (range 43-81); $66 \%$ male). All patients had disease proven by non-cardiac biopsy. Cardiac involvement was ascertained through echocardiography, supported by a Mayo clinic classification score of 2 or 3.1718

6. Infarct zone in myocardial infarction $(n=20$; median age 58 (range 41-72); 90\% male). EQ-CMR was performed within 1 week of an acute myocardial infarction. The infarct zone was identified by late gadolinium enhancement (50\% following a culprit lesion in the left anterior descending artery, 35\% right coronary and $15 \%$ circumflex artery) with the myocardial T1 measurements taken from this area.

Patients with atrial fibrillation or a contraindication to contrast CMR examination were excluded from the study.

All patients were recruited from tertiary clinical and research departments at the Heart Hospital (AS and myocardial infarction) or affiliated national centres (Inherited Cardiac Disease Unit, Heart Hospital (HCM, DCM and AFD) and National Amyloidosis Centre, Royal Free Hospital).

Analysis of T1 was as previously described ${ }^{14}$; myocardial T1 was measured with a region of interest covering the basal and mid septum (figure 1) avoiding areas of late gadolinium

\begin{tabular}{|c|c|c|c|c|}
\hline Variable & All & Male & Female & p Value \\
\hline Number & 81 & 42 & 39 & NS \\
\hline Age (years), median (range) & $43(24-81)$ & $36(24-81)$ & $46(24-68)$ & NS \\
\hline Weight (kg) & $76 \pm 13$ & $82 \pm 11$ & $70 \pm 11$ & $<0.001$ \\
\hline Height $(\mathrm{cm})$ & $171 \pm 10$ & $178 \pm 8$ & $165 \pm 7$ & $<0.001$ \\
\hline Haematocrit & $0.42 \pm 0.03$ & $0.43 \pm 0.03$ & $0.40 \pm 0.02$ & $<0.001$ \\
\hline eGFR $\left(\mathrm{ml} / \mathrm{min} / 1.73 \mathrm{~m}^{2}\right)$ & $93 \pm 15$ & $94 \pm 14$ & $93 \pm 16$ & NS \\
\hline Heart rate (beats/min) & $63 \pm 10$ & $60 \pm 8$ & $66 \pm 10$ & $<0.01$ \\
\hline $\mathrm{EDV}_{\mathrm{i}}\left(\mathrm{ml} / \mathrm{m}^{2}\right)$ & $73 \pm 13$ & $78 \pm 12$ & $67 \pm 11$ & $<0.001$ \\
\hline $\mathrm{ESV}_{\mathrm{i}}\left(\mathrm{ml} / \mathrm{m}^{2}\right)$ & $24 \pm 7$ & $27 \pm 7$ & $21 \pm 6$ & $<0.001$ \\
\hline $\mathrm{EF}(\%)$ & $67 \pm 5$ & $66 \pm 5$ & $70 \pm 5$ & $<0.001$ \\
\hline Mass $_{\mathrm{i}}\left(\mathrm{g} / \mathrm{m}^{2}\right)$ & $67 \pm 13$ & $75 \pm 11$ & $57 \pm 8$ & $<0.001$ \\
\hline $\mathrm{LAA}_{\mathrm{i}}\left(\mathrm{cm}^{2} / \mathrm{m}^{2}\right)$ & $11 \pm 2$ & $11 \pm 2$ & $11 \pm 2$ & NS \\
\hline Septal maximal wall thickness (mm) & $6.8 \pm 1$ & $7.6 \pm 1$ & $6.0 \pm 1$ & $<0.001$ \\
\hline
\end{tabular}

Table 1 Baseline characteristics of normal volunteers

Values are mean \pm SD unless stated.

$E D V_{i}$, left ventricular end diastolic volume indexed; $E F$, left ventricular ejection fraction; eGFR, estimated glomerular filtration rate calculated using the modification of diet in renal disease equation; $E_{\mathrm{i}} \mathrm{V}_{\mathrm{i}}$ left ventricular end systolic volume indexed; $\mathrm{LAA}_{\mathrm{i}}$, left atrial area indexed; Mass ${ }_{i}$, left ventricular mass indexed; NS, no significant correlation. 
Figure 1 (A) Typical regions of interest used in this study in the basal to mid septum and descending aorta in a normal subject, here from a fast lowangle single-shot inversion recovery image taken at an inversion time of 600 ms at contrast equilibrium. (B) Equivalent image from a patient with Anderson-Fabry disease (AFD) showing the regions of interest used for the study in the basal to mid septum and descending aorta to assess the extracellular volume (ECV) in potential diffuse fibrosis (in this case the ECV was in the normal range). Late enhancement is indicated by an arrow
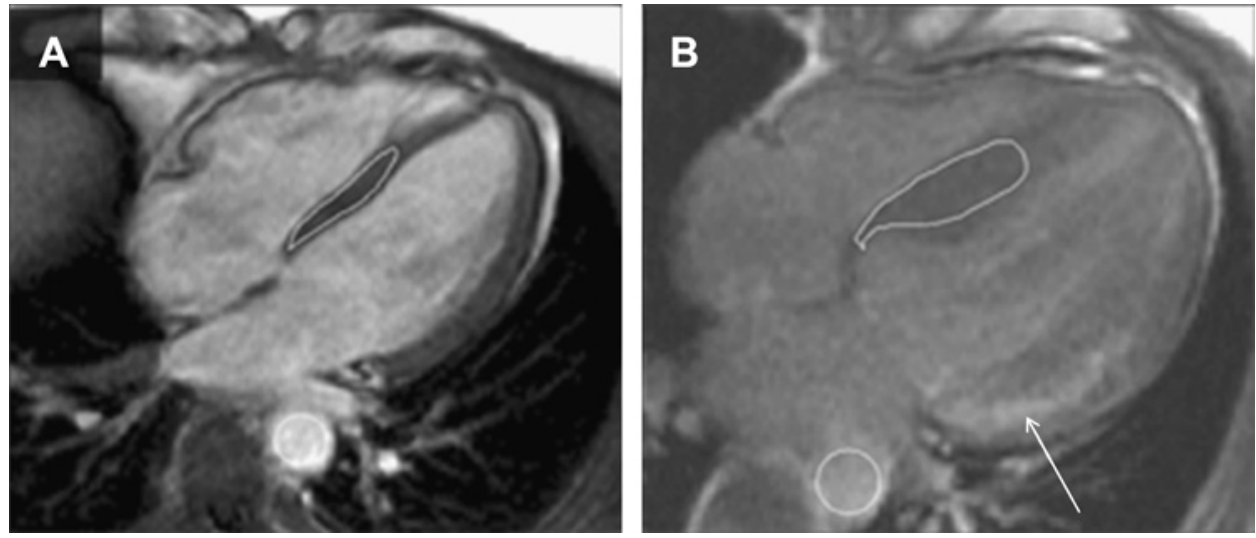
in the basal to mid lateral wall showing the presence of focal fibrosis (seen in this area in $\sim 50 \%$ of patients with AFD). This shows the heterogeneity in amount and type of scar in AFD, which has previously been found on necropsy ${ }^{19}$ and is further illustrated by our equilibrium contrast cardiovascular magnetic resonance data.

enhancement (which represent focal, rather than diffuse fibrosis), except in the patients with myocardial infarction, where the infarct zone was assessed. Areas of late enhancement were not avoided in cardiac amyloidosis, as in this disease it does not represent focal fibrosis and is ill-defined. The basal and mid septum were assessed, as the FLASH IR method is a multibreath-hold one precluding whole heart analysis. The blood T1 was assessed in the descending aorta.

Results were analysed by DS, AF and JM using SPSS (V.19). All patients and normal subjects studied were included in the statistical analysis. ECV values were found to be normally distributed in each group (Kolmogorov-Smirnov test, $p>0.05$ for each dataset) and so expressed as mean \pm SD. Results in different subgroups were compared either using a Student t test or, in multiple groups, by one-way analysis of variance with post-hoc analysis using the Games-Howell procedure because of different variances. Correlations were evaluated using Pearson coefficients.

\section{RESULTS}

The mean myocardial ECV in normal subjects was $0.253 \pm 0.035$ and was higher in women than men $(0.273 \pm 0.029$ vs $0.233 \pm 0.029, \mathrm{p}<0.001$; figure 2$)$. This did not change either with gender or overall with age $(R=0.09, p=0.42)$. There was a correlation of myocardial ECV with height $(R=0.42$, $\mathrm{p}<0.0001)$ and haematocrit $(\mathrm{R}=0.39, \mathrm{p}=0.005)$ in all subjects, but, when split by gender, haematocrit correlated in women $(\mathrm{R}=0.36, \mathrm{p}=0.03)$ but not men $(\mathrm{R}=0.01, \mathrm{p}=0.96)$.

In patients, the myocardial ECV of the intracellular storage disorder, AFD, was the same as in normal subjects $(0.250 \pm 0.023$ vs $0.253 \pm 0.035, p=0.99)$. In every other disease, myocardial ECV was higher $(p<0.001$ total; all pair-wise comparisons with normal myocardium $\mathrm{p}<0.03)$. There was no difference in

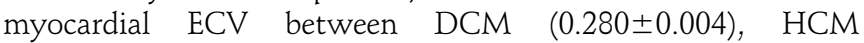
$(0.291 \pm 0.005)$ and AS $(0.276 \pm 0.044, p=N S)$, but myocardial ECV in cardiac AL amyloidosis $(0.466 \pm 0.070)$ was higher than in these three diseases $(p<0.001)$, and infarct zone in myocardial infarction $(0.585 \pm 0.076)$ was higher than in cardiac $\mathrm{AL}$ amyloidosis $(p<0.001)$ (figure 3$)$. When patient groups were compared with gender-matched normal subjects rather than the whole normal control population, all statistical testing remained highly significant except for AFD which remained non-significant.

The correlations seen between myocardial ECV and other clinical CMR parameters are shown in table 2. In patients with
AFD, no correlations were found. In other diseases, significant correlations were found with ejection fraction (cardiac AL amyloidosis, $\mathrm{R}=0.57, \mathrm{p}=0.005$; $\mathrm{DCM}, \mathrm{R}=0.35$, $\mathrm{p}=0.03$ ), indexed left ventricular systolic volume (cardiac $A L$ amyloidosis, $R=0.44$, $\mathrm{p}<0.001$; AS, $\mathrm{R}=0.51, \mathrm{p}=0.04$ ), indexed left ventricular mass (cardiac AL amyloidosis, $\mathrm{R}=0.44, \mathrm{p}=0.04 ; \mathrm{DCM}, \mathrm{R}=-0.36$, $\mathrm{p}=0.005)$, indexed left atrial area (DCM, $\mathrm{R}=0.65, \mathrm{p}<0.001)$, aortic valve area (AS, $\mathrm{R}=-0.41, \mathrm{p}=0.001$ ), percentage of late gadolinium enhancement (HCM, R=0.48, $\mathrm{p}=0.05$ ) and septal thickness (cardiac AL amyloidosis, $\mathrm{R}=0.51, \mathrm{p}=0.005$ ).

\section{DISCUSSION}

Using the new test, EQ-CMR, we measured the myocardial extracellular volume, ECV, non-invasively in the basal to mid septum in health and disease. Results from disease states were found to be different from in normal subjects, with the single exception of AFD - the example of an intracellular (lysosomal) storage disease ${ }^{20}$-where no increase in myocardial ECV occurred. In cardiac AL amyloidosis, in which the heart is well recognised to be subject to widespread and substantial extracellular infiltration, ${ }^{17}$ the myocardial ECV was larger than in all other diseases apart from myocardial infarct zone, where the myocardial ECV was the highest. Correlations of myocardial ECV with cardiac variables were noted, but further work is

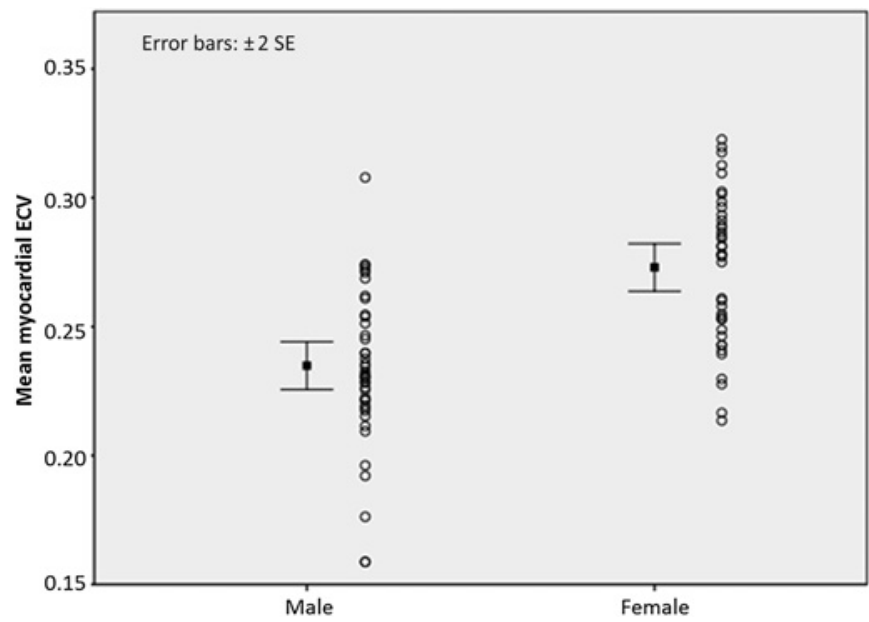

Figure 2 Male versus female extracellular volume (ECV; mean \pm 2 standard errors and dot plots) in normal subjects. 
Figure 3 Extracellular volume (ECV) in health and disease: inter-group comparisons. The same data are presented as mean \pm 2 standard errors (A) and a dot plot (B).
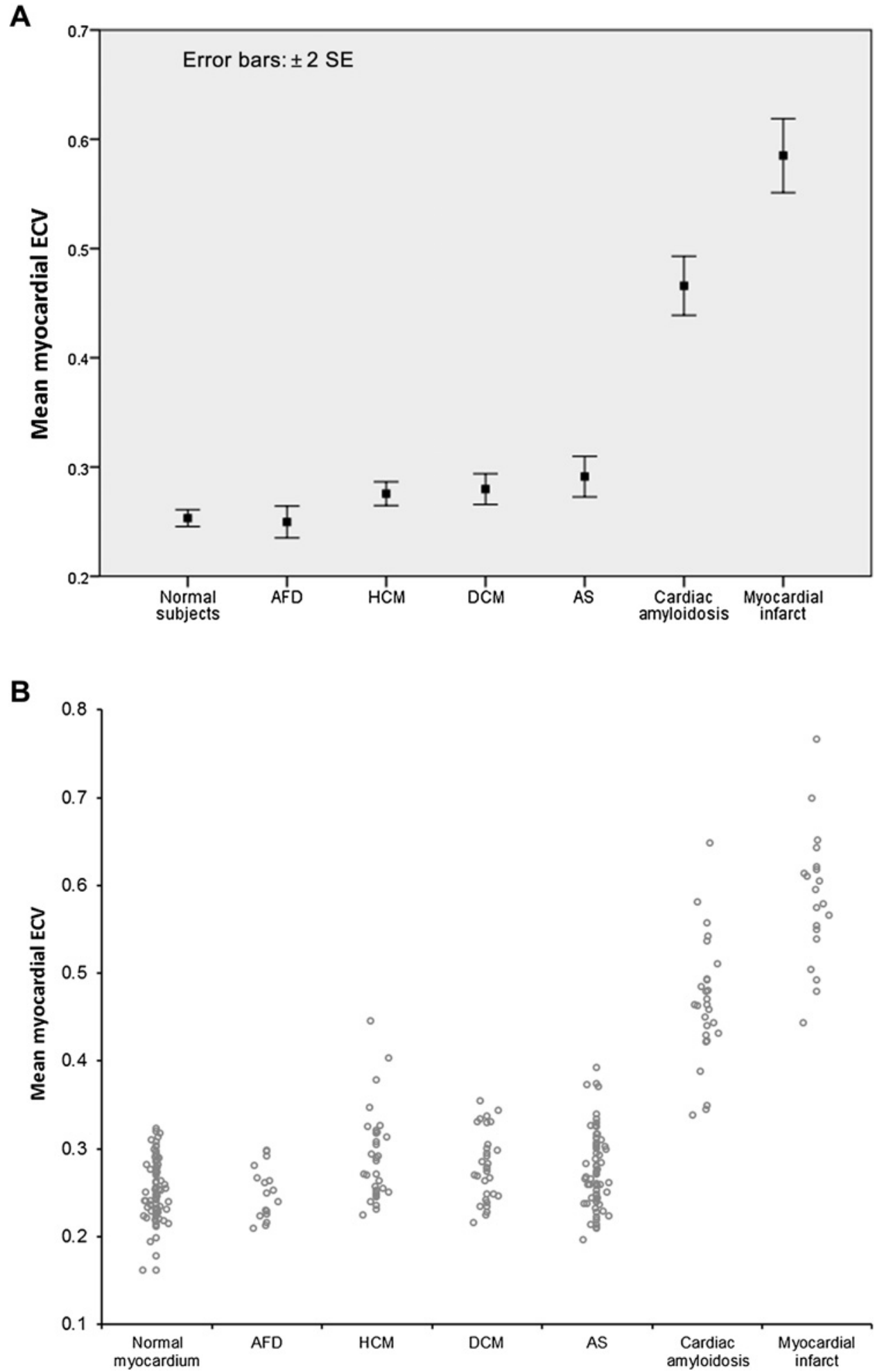

needed to assess these in more detail. In addition, normal subjects were shown to have no increase in myocardial ECV with age, but women had higher myocardial ECV than men. Further work is required to assess and understand the clinical and pathophysiological significance of these findings.

EQ-CMR uses a standard MRI scanner and a routine clinical contrast agent in a new way to quantify the ECV, a fundamental property of organs and tissues. It is rarely possible to measure this variable directly by myocardial biopsy either because of sampling error in disease or inadequate risk/benefit ratio in health. EQ-CMR overcomes this problem by safely and noninvasively sampling a larger volume, or potentially the whole organ (with newer T1 mapping sequences), without exposure to ionising radiation. Although we present findings on assessment of the myocardium, the technique could also potentially be used to evaluate other organs, as the induced contrast equilibrium occurs throughout the body.

In this study, two diseases stand out statistically-AFD and cardiac AL amyloidosis. AFD is a rare X-linked inherited condition, which occurs as a result of mutations in the GLA gene. ${ }^{20}$ 
Table 2 Pearson correlations between extracellular volume and other cardiac magnetic resonance clinical variables

\begin{tabular}{|c|c|c|c|c|c|c|}
\hline Disease & EF & $\mathrm{EDV}_{\mathrm{i}}$ & ESV $_{\mathrm{i}}$ & Mass $_{i}$ & $\mathrm{LAA}_{\mathrm{i}}$ & Other \\
\hline Normal subjects & NS & NS & NS & $\mathrm{R}=-0.36^{* *}$ & NS & None \\
\hline AFD & NS & NS & NS & NS & NS & None \\
\hline DCM & $\mathrm{R}=0.35^{*}$ & NS & NS & $R=-0.36^{*}$ & $\mathrm{R}=0.65^{* * *}$ & None \\
\hline HCM & NS & NS & NS & NS & NS & $\%$ LGE: $\mathrm{R}=0.48^{*}$ \\
\hline Cardiac amyloidosis & $\mathrm{R}=-0.57^{* *}$ & NS & $\mathrm{R}=0.63^{* * *}$ & $\mathrm{R}=0.44^{*}$ & NS & Septal thickness: $\mathrm{R}=0.51^{*}$ \\
\hline
\end{tabular}

NS, No significant correlation; ${ }^{*} \mathrm{p}<0.05 ;{ }^{* *} \mathrm{p}<0.01$; ${ }^{* * *} \mathrm{p}<0.001$.

AFD, Anderson-Fabry disease; AS, aortic stenosis; AVA, aortic valve area; DCM, dilated cardiomyopathy; HCM, hypertrophic cardiomyopathy; LGE, late gadolinium enhancement.

This results in deficiency of $\alpha$ galactosidase A, leading to accumulation of glycosphingolipid inside cells. The most common cardiac manifestation is left ventricular hypertrophy. In our cohort, $76 \%$ of the patients were male, of whom $86 \%$ had left ventricular hypertrophy. All female patients had left ventricular hypertrophy. In recent years, there has been a focus on extracellular expansion in AFD, as $\sim 50 \%$ of patients show late gadolinium enhancement ( $53 \%$ in this study), which is likely to represent focal fibrosis, particularly in the basal inferolateral wall of the left ventricle. ${ }^{21}$ Previous necropsy work in three hearts similarly found macroscopic fibrosis in this area ${ }^{19}$ but only limited microscopic septal fibrosis (1\% septal vs $17 \%$ lateral wall). This is in keeping with our results and suggests that the fibrosis pattern in AFD is heterogeneous. It is a limitation of this study that whole heart quantification was not performed.

Cardiac amyloidosis is a disease known to cause extracellular expansion. ${ }^{17}$ At autopsy, the amyloid burden may be the majority of the heart by volume. Here we studied the AL variant, where amyloid is secondary to amyloidogenic serum light chains from a plasma cell dyscrasia. Cardiac involvement is associated with a poor prognosis due to cardiac failure and arrhythmia, but direct amyloid involvement in the heart is not currently measurable without biopsy. In this study, the amyloid myocardial ECV distribution showed no overlap with normal subjects (the lowest cardiac amyloid myocardial ECV being greater than the highest normal myocardial ECV), suggesting potential diagnostic and quantitative utility as a test for cardiac amyloid burden.

In this preliminary study, correlations were found between myocardial ECV and CMR parameters of cardiac size, mass and function. The range of these correlations and their heterogeneity suggests the possibility of disease-specific patterns. Further work is needed to explore these associations. Correlations of myocardial ECV with blood biomarkers, particularly collagen biomarkers, and diastolic function are currently unknown, as is its relationship to patient morbidity and mortality.

In this study there was no correlation in normal subjects between age and myocardial ECV. Although animal work suggests an age-related increase in left ventricular fibrosis and hence extracellular expansion, ${ }^{22}$ other lines of evidence are conflicting. Human autopsy studies have been performed. One small study $(n=12)$ suggests an increase in left ventricular fibrosis with age ${ }^{23}$; another $(n=230)$ showed no such change in the LV conduction system, ${ }^{24}$ and another $(n=67)$ suggested the reverse- - decrease in left ventricular myocardial ECV, and an increase in the myocyte volume percentage with age. ${ }^{25}$ In the CMR literature, a recent study by Ugander et al using a similar method to the one described in the present study showed a weak correlation between age and myocardial ECV in 60 individuals $(\mathrm{R}=0.28, \mathrm{p}=0.01){ }^{26}$ The individuals were those with a normal CMR scan rather than healthy volunteers, and there was a high rate of comorbidity (53\% hypertensive, 10\% diabetic), which may increase myocardial ECV. ${ }^{27-29}$ Blood biomarker studies of collagen turnover have found no, or only weak, correlations with age in normal subjects. ${ }^{30} 31$

Another finding in this study was that normal female subjects had a myocardial ECV 0.04 times higher than male subjects-a 1.4 SD difference. The dataset was not designed to assess this unexpected finding in detail, but four possible explanations can be suggested. The first is that it is a consequence of the measurement technique due to partial voluming. Theoretically, the thinner female septum will be more prone to this problem (pre-contrast myocardial T1 increased; post-contrast myocardial T1 reduced), resulting in an apparently higher myocardial ECV (and potentially a negative correlation between ECV and left ventricular mass index, as found). This effect would confound the male versus female variation described in this study but also reinforce our findings of differences between health and disease, as partial voluming occurs less in hypertrophied septum.

The second possible explanation is differences in gender haematocrits. The myocardial ECV includes water between myocytes and within capillaries between red cells. Anaemia will expand the latter; however, we note a female but not male correlation between haematocrit and myocardial ECV, despite similar haematocrit ranges.

The third possible explanation is that the finding merely reflects a non-cardiovascular systematic male-female difference, such as in height or weight, and thus a smaller heart or faster heart rate. However, this explanation begs the question as to why cardiac scaling would cause altered myocardial ECV, and makes the false supposition of independence of cardiovascular function from body size.

The fourth possible explanation is that women do indeed have more extracellular expansion for a cardiovascular reason. This would potentially contribute to explanations of male/ female differential disease expression, ${ }^{32}$ fitting with known male/female differences in systemic and cardiac macro- and micro-vasculature structure and function, ${ }^{33}$ with potential contributions from the differential effects of sex hormones on myocardium. ${ }^{34}$ Using our data, a multivariate analysis suggests that the only two independent predictors of myocardial ECV elevation in normal people were gender and RR interval- that is, haematocrit, indexed mass, height and septal thickness were not predictors. However, this result needs to be interpreted with caution. Newer single-breath-hold T1 mapping techniques with higher resolution and motion correction are being developed, which will minimise any partial voluming effects and therefore allow us to revisit this question.

The technique used here, EQ-CMR, is in the current iteration, an add-on to a standard clinical scan, but as implemented, has limitations. The FLASH IR T1 mapping sequence is robust, transferable to other MRI scanners, and has been shown to allow accurate assessment of myocardial ECV against biopsy, but is not fast and does not allow whole-heart assessment. 
Although the use of a contrast infusion to achieve equilibrium is likely to be the gold standard method for this technique, as it eliminates contrast kinetic issues, it is also time consuming. Therefore newer T1 mapping sequences ${ }^{35} 36$ and dynamic equilibrium $^{37}$ (achieved after only a bolus administration of contrast) are likely to increase the ease and clinical applicability of the technique and will allow whole-heart quantification.

In conclusion, myocardial ECV, assessed non-invasively in the septum with EQ-CMR, shows small gender differences in normal individuals and disease-specific changes which correlate with CMR parameters of disease severity. These results and the range of potential technical improvements make the non-invasive measurement of myocardial ECV by CMR a promising area for future research and a potentially useful clinical tool across a range of indications.

Contributors DMS: led study and four patient groups; lead writer of manuscript. ASF, SMB, SKW: each contributed a patient group. VM, GQ: scanning, recruitment, analysis. $\mathrm{RH}, \mathrm{EM}, \mathrm{AM}, \mathrm{DAH}, \mathrm{WJM}, \mathrm{PME}, \mathrm{DJH}, \mathrm{PNH}$ : clinicians advising and coordinating patient cohorts. AMT: senior CMR author; role in optimising the clinical and research CMR techniques used and then in the writing and formatting of the manuscript. JCM: concept and design of the study. All authors helped with writing, formatting and reviewing of manuscript.

Funding (1) British Heart Foundation; (2) Genzyme (for the Anderson-Fabry arm of the study).

Competing interests RHL: received honoraria, consulting fees or grant funding from Genzyme, Shire and Amicus. DAH: travel, research support and honoraria for speaking and being on advisory boards from Shire, Genzyme and Glaxo SmithKline/Amicus. EM: unrestricted educational grants, travel expenses and honoraria from Shire and Genzyme. PME: consulting fees for Shire and Genzyme and speaker fees for Shire.

Provenance and peer review Not commissioned; externally peer reviewed.

\section{REFERENCES}

1. Klima M, Burns TR, Chopra A. Myocardial fibrosis in the elderly. Arch Pathol Lab Med 1990;114:938-42.

2. Wynn TA. Cellular and molecular mechanisms of fibrosis. J Pathol 2008;214:199-210.

3. Mewton N, Liu CY, Croisille $P$, et al. Assessment of myocardial fibrosis with cardiovascular magnetic resonance. J Am Coll Cardiol 2011;57:890-903.

4. Pepys MB. Amyloidosis. Annu Rev Med 2006;57:223-41.

5. Lewis J, Mohanty S. Nonalcoholic fatty liver disease: a review and update. Dig Dis Sci 2010;55:560-78.

6. Araya J, Nishimura S. Fibrogenic reactions in lung disease. Annu Rev Pathol Mech Dis 2010;5:77-98.

7. Guido M, Rugge M. Liver biopsy sampling in chronic viral hepatitis. Semin Liver Dis 2004:24:89-97.

8. Nair V, Butany J. Heart transplant biopsies: interpretation and significance. J Clin Pathol 2010;63:12-20.

9. Hawkins PN, Pepys MB. Imaging amyloidosis with radiolabelled SAP. Eur J Nucl Med 1995;22:595-9.

10. Holzmann M, Nicko A, Kuhl U, et al. Complication rate of right ventricular endomyocardial biopsy via the femoral approach: a retrospective and prospective study analyzing 3048 diagnostic procedures over an 11-year period. Circulation 2008;118:1722-8.

11. Kim RJ, Wu E, Rafael A, et al. The use of contrast enhanced magnetic resonance imaging to identify reversible myocardial dysfunction. N Engl J Med 2000;343:1445-53.

12. Arheden H, Saeed M, Higgins CB, et al. Measurement of the distribution volume of gadopentetate dimeglumine at echo-planar MR imaging to quantify myocardial infarction: comparison with ${ }^{99 \mathrm{~m}} \mathrm{Tc}$-DTPA autoradiography in rats. Radiology 1999;211:698-708.
13. Jerosch-Herold M, Sheridan DC, Kushner JD, et al. Cardiac magnetic resonance imaging of myocardial contrast uptake and blood flow in patients affected with idiopathic or familial dilated cardiomyopathy. Am J Physiol Heart Circ Physiol 2008:295:H1234-42.

14. Flett AS, Hayward MP, Ashworth MT, et al. Equilibrium contrast cardiovascular magnetic resonance for the measurement of diffuse myocardial fibrosis: preliminary validation in humans. Circulation 2010;122:138-44.

15. Mestroni L, Maisch B, McKenna WJ, et al. Guidelines for the study of familial dilated cardiomyopathies. Eur Heart J 1999:20:93-102.

16. Elliott P, Andersson B, Arbustini E, et al. Classification of the cardiomyopathies: a position statement from the European Society of Cardiology Working Group on Myocardial and Pericardial Diseases. Eur Heart J 2008;29:270-6.

17. Falk RH, Dubrey SW. Amyloid heart disease. N Engl J Med 1997;33:898-909.

18. Dispenzieri A, Gertz MA, Kyle RA, et al. Serum cardiac troponins and N-terminal pro-brain natriuretic peptide: a staging system for primary systemic amyloidosis. $J$ Clin Oncol 2004;22:3751-7.

19. Sheppard MN, Cane P, Florio R, et al. A detailed examination of heart tissue from three older patients with Anderson-Fabry disease on enzyme replacement therapy. Cardiovasc Pathol 2010;19:293-301.

20. O'Mahony C, Elliott P. Anderson-Fabry disease and the heart. Prog Cardiovasc Dis 2010:52:326-35

21. Moon JC, Sachdev B, Elkington AG, et al. Gadolinium enhanced CMR in AndersonFabry disease: evidence for a disease specific abnormality of the myocardial interstitium. Eur Heart J 2003;24:2151-5.

22. Kwak HB, Kim JH, Joshi $K$, et al. Exercise training reduces fibrosis and matrix metalloproteinase dysregulation in the aging rat heart. FASEB $\mathrm{J}$ 2011;25:1106-17.

23. Gazotti Debessa CR, Mesiano Maifrino LB, Rodrigues de Souza R. Age related changes of collagen network of the human heart. Mech Aging Devel 2001;122:1049-58

24. Song $\mathbf{Y}$, Yao 0 , Zhu J, et al. Age-related variation in the extracellular tissues of the cardiac conduction system; and autopsy study of 230 Han Chinese. For Sci Int 1999;104:133-42.

25. Olivetti G, Melissari M, Capasso JM, et al. Cardiomyopathy of the aging human heart. Myocyte loss and reactive cellular Hypertrophy. Circ Res 1991:68:1560-8

26. Ugander M, Oki AJ, Hsu LY, et al. Extracellular volume imaging by magnetic resonance imaging provides insights into overt and sub-clinical myocardial pathology. Eur Heart J 2012:33:1268-78.

27. Jellis C, Wright J, Kennedy D, et al. Association of imaging markers of myocardia fibrosis with metabolic and functional disturbances in early diabetic cardiomyopathy. Circ Cardiovasc Imaging 2011;4:693-702.

28. Jabbour A, Ismail TF, De Noronha SV, et al. Non-invasive assessment of interstitial myocardial fibrosis in pressure-overload left ventricular hypertrophy. J Cardiovasc Magn Reson 2012;14(Suppl 1):05.

29. Mongeon FP, Jerosch-Herold M, Coelho-Filho OR, et al. Identification of myocardia extracellular matrix expansion by cardiac MRI in hypertensive patients. J Cardiovasc Magn Reson 2011:13(Suppl 1):0109.

30. Barasch E, Gottdiener JS, Aurigemma G, et al. Association between elevated fibrosis markers and heart failure in the elderly: the cardiovascular health study. Circ Heart Fail 2009;2:303-10

31. Melkko J, Niemi S, Risteki L, et al. Radioimmunoassay of the carboxyterminal propeptide of human type 1 procollagen. Clin Chem 1990;36:1328-32.

32. Hayward CS, Kelly RP, Collins P. The roles of gender, the menopause and hormone replacement on cardiovascular function. Cardiovasc Res 2000:46:28-49.

33. Shaw LJ, Merz CN, Pepine CJ, et al; WISE Investigators. Insights from the NHLBI-Sponsored Women's Ischemia Syndrome Evaluation (WISE) Study: Part I: gender differences in traditional and novel risk factors, symptom evaluation, and gender-optimized diagnostic strategies. J Am Coll Cardiol 2006;47 (3 Suppl):4-S20.

34. Regitz-Zagrosek V, Seeland U Sex and gender differences in myocardia hypertrophy and heart failure. Wien Med Wochenschr 2011;161:109-16.

35. Piechnik SK, Ferreira VM, Dal'Armelina E, et al. Shortened Modified Look-Locker inversion recovery (ShMOLLI) for clinical myocardial T1-mapping at 1.5 and 3T within a 9 heartbeat breathhold. J Cardiovasc Magn Reson 2010;12:69.

36. White SK, Sado DM, Flett AS, et al. Characterising the myocardial interstitial space: the clinical relevance of non-invasive imaging. Heart 2012;98:773-9.

37. Schelbert EB, Testa SM, Meier CG. Myocardial extravascular extracellular volume fraction measurement by gadolinium cardiovascular magnetic resonance in humans: slow infusion versus bolus. J Cardiovasc Magn Reson 2011;13:16. 